\title{
APUNTES SOBRE LA INJURIA EN LAS XII TABLAS Y SU TRANSMISIÓN TEXTUAL*
}

\author{
NOTES REGARDING THE NATURE OF PERSONAL INJURIES IN THE \\ LAW OF THE TWELVE TABLES AND ITS TEXTUAL TRANSMISSION
}

\author{
Patricio-Ignacio CarvajaL ${ }^{* *}$
}

La Ley de las XII Tablas (451-450

a. C.), convencionalmente contiene en su Tabla 8 la regulación arcaica de los distintos delitos que en época clásica serán conocidos como injuria:

\section{SI MEMBRUM RUPSIT, NI CUM EO PACIT, TALIO ESTO. \\ 3. SI OS FREGIT LIBERO, CCC, SI SERVO, CL POENAM SUBITO. \\ 4. SI INIURIAM ALTERI FAXSIT, VIGINTI QUINQUE POENAE SUNTO.}

\section{Traducción}

2. Si rompe un miembro, y no pacta con él, habrá talión.

3. Si fractura un hueso en un libre, habrá una pena de 300 (ases), si en un esclavo, de 150.

4. Si hace injuria a otro, habrá veinticinco (ases) como pena.

\footnotetext{
* El presente trabajo forma parte del proyecto Fondecyt Regular No 1095220, del proyecto Fondecyt Regular No 1100452 y del Proyecto Anillo de Investigación Asociativa-CONICYT, código SOC 1111.

** Profesor de Derecho Romano. Pontificia Universidad Católica de Chile. Licenciado de Derecho de la Pontificia Universidad Católica de Chile. Doctor en Derecho por la Universidad de Salamanca. Correo electrónico: carvajal@uc.cl.
}

Para formarse este cuadro, la doctrina romanista ${ }^{1}$ ha tenido frente a sí una serie

1 Voigt, M. (1883): Die XII Tafeln Geschichteund System des Civil- und Criminal-Rechtes, wie-Processes der XII Tafelnnebstderen Fragmenten, 2 vols. (Leipzig, Verlag von A. G. Liebeskind); Huvelin, P. (1903): La notion de l'iniuria dans le très ancien droit romain, Mélanges Ch. Appleton (Lyon, A. Rey, imprimeuréditeur ); Binding, K. (1919): "Rechtsvergleichende Vermutungen zu membrum ruptum, os fractum und iniuria der lex XII Tabularum”, ZSS 40, pp. 106-112; Appleton, Ch. (1926): "Nôtre einsegnement du droit romain. Ses ennemis et ses défauts", en Mélanges de Droit Romain dé diés à Georges Cornil, 1 (Paris, Sirey) pp. 43-79 (esp. pp. 51 ss.); Luzzato, G.-I. (1934): Per unipotesi sulle origini e la natura delle obbligazioni romane (Milano, Dott. A. Giuffrè) pp. 173 ss.; Pugliese, G. (1940): Studi sull'iniuria, 1 (Milano, Dott. A. Giuffrè) pp. 1 ss.; Di Paola, S. (1946-7): "La genesi storica del delitto di iniuria", en Annali del Seminario Giuridico dell'Università di Catania, 1, pp. 268 ss.; KASER, M. (1949): Das altrömische Ius. Studien zur Rechtsvorstellungund Rechtsgeschichte der Römer (Göttingen, Vandenhoeck und Ruprecht) pp. 207 ss.; Simon, D. (1965): "Begriffund Tatbestand der "iniuria" in altrömischen Recht", ZSS 82, pp. 132-187; Da Nóbrega, V. (1967): "L'iniuria dans la loi des XII Tables”, Romanitas 8, pp. 250-279; BIRKs, P. (1969): "The early history of iniuria", $T v R 37$, pp. 163-208; v. LÜвтоw, U. (1969): “Zum römischen Injurienrecht”, Labeo 15, pp. 131-167; KASER, M. $\left(1971^{2}\right)$ : Das römische Privatrecht, 1 (München, C. H. Beck'sche Verlgasbuchhandlung) pp. 156 y 157; Wittmann, R. (1972): Körperverletzung an Freien im klassischen römischen Recht (München, C. H. Beck) pp. 3 ss.; Watson, A. (1975): "Personal injuries in the XII Tables”, $T v R$ 43, pp. 213-222; Halpin, A. K. W. (1976): "The usage of iniuria in the Twelve Tables", The Irish Jurist 11, pp. 344-354; Manfredini, A. (1977): Contributi allo studio dell'“iniuria” in età repubblicana (Milano, Dott. A. Giuffrè) pp. 15 ss.; 
de fuentes jurídicas y extrajurídicas. Entre las jurídicas, ciertamente es fundamental el testimonio de Gayo, Inst.223 y 224:

Poena autem iniuriarum ex lege XII tabularum propter membrum quidem ruptum talio erat; propter os uero fractum

Plescia, J. (1977): "The development of "iniuria"”, Labeo 23, pp. 271-289; Pugliese, G. (1978): "Rec. Manfredini, Contributi allo studio dell' "iniuria" in età repubblicana”, IVRA, pp. 193-206; GIofFREDI, C. (1980): "In tema di 'iniuria' (sui fattori di formazione del diritto romano in età preclassica)", en Nuovi Studi di Diritto Greco e Romano, Romae, Pontificia Universitas Lateranensis, pp. 145-172; VölKL, A. (1984): Die Verfolgung der Körperverletzung im frühen römischen Recht (Wien-Koln-Gratz, Böhlau) pp. 16 ss., 40 ss. y 144 ss.; Pólay, E. (1984): "Iniuria-Tatbestände im archaischen Zeitalter des antiken Rom”, ZSS 101, pp. 142-189; Pólay, E. (1986): Iniuria Types in Roman law (Budapest, Akadémiai Kiadó) pp. 16 ss.; Westbrook, R. (1988): "Nature and origins of the Twelve Tables", ZSS 105, pp. 103 ss. (pp. 74-121); Rodger, A. (1991): "Introducing iniuria”, TvR 59, pp. 1-11; Bassanelli Sommariva, G. (1995): "Note sulla parola poena in XII Tab. 8.3 e 8.4", en Studi in Onore di Enrico Bassanelli, E. Casadei; G. Sgarbenti (a cura di), (Milano, Giuffrè)pp. 793-816; Zimmermann, R. (1996): The law of obligations. Roman foundations of the civilian tradition (Oxford, University Press) pp. 1050 a 1053; Bignardi, A. (1997): "Frangere e rumpere nel lessico normativo e nella interpretatio prudentium", en Nozione, Formazione e Interpretazione del Diritto. Dall'età romana alle esperienze moderne. Ricerche dedicate al Professor Filippo Gallo, 1 (Napoli, Jovene) pp. 11-60; Santalucia, B. (19982): Diritto e proceso penale nell'antica Roma (Milano, Dott. A. Giuffrè) pp. 60 y 61; Hagemann, M. (1998): Iniuria. Von den XII-Tafeln bis zur justinianischen Kodifikation (Köln-Weimar-Wien, Böhlau); Cursı, M. F. (2002): Iniuria cum damno. Antigiuridicità e colpevolezza nella storia del danno aquiliano (Milano, Dott. A. Giuffrè Editore) pp. 221 a 268; Cursi, M. F. (2010): Danno e responsabilità extracontrattuale nella storia del diritto privato (Napoli, Jovene Editore) pp. 7 a 19; Bassanelli Sommariva, G. (2012): Lezioni di diritto privato romano, 3 (Santarcangelo di Romagna, Maggioli Editore) pp. 260 a 268; GuZmán BRito, A. $\left(2013^{2}\right)$ : Derecho privado romano, 2 (Santiago, Legal Publishing-Thomson Reuters) pp. 302 y 303. aut conlisum trecentorum assium poena erat, si libero os fractum erat; at si seruo, CL; propter ceteras uero iniurias XXV assium poena erat constituta. Et uidebantur illis temporibus in magna paupertate satis idoneae istae pecuniariae poenae.

224. Sed nunc alio iure utimur. Permittitur enim nobis a praetore ipsis iniuriam aestimare, et iudex uel tanti condemnat, quanti nos aestimauerimus, uel minoris, prout ei uisum fuerit...

\section{Traducción}

Pero la pena por la injuria según la ley de las XII Tablas por un miembro ciertamente roto era el talión; en cambio, por un hueso fracturado o trizado la pena era de 300 ases, si el hueso era fracturado a un libre; y si a un esclavo, 150; en cambio, para las demás injurias la pena establecida era de 25 ases. Y perecían en aquellos tiempos de gran pobreza suficientemente idóneas estas penas pecuniarias.

224. Pero ahora usamos otro derecho. Ciertamente se permite por el pretor estimar la injuria por nosotros mismos, y el juez o condena a tanto cuanto nosotros hayamos estimado, o a menos, según fuera el parecer de este...

Además de esta obra institucional, también tiene la máxima relevancia Paulo, libro singulari et titulo de iniuriis, Collatio legum Mosaicarum et Romanarum 2.5.4 y 5:

...Iniuriarum actio aut legitima est aut honoraria. 5. Legitima ex lege duodecim tabularum: 'qui iniuriam alteri facit, quinque et uiginti sestertiorum poenam subito'. Quae lex generalis fuit: fuerunt et speciales, uelut illa: 'si os fregit libero, CCC si seruo, CL poenam subito sestertiorum: 


\section{Traducción}

...La acción de las injurias o es legítima o es honoraria. 5. Legítima por la ley de las XII Tablas: 'quien hace injuria a otro, sufrirá la pena de veinticinco ases'. La cual fue una ley general: hubo también especiales, como aquella: 'si fractura un hueso a un libre, de 300 si a un esclavo, de 150 sestercios sufrirá una pena'.

A esto se debe añadir los testimonios de las Pauli Sententiae 5.4.6 y $7^{2}$ y de Justiniano, Inst. $4.4 .7^{3}$.

2 Iniuriarum actio aut lege aut more aut mixto iure introductanest. Lege duo decim tabularum de famosis carminibus, membris ruptis et ossibus fractis. 7. Moribus, quotiens factum pro qualitate sui arbitrio iudicis aestimatur, congruentis poenae supplicio vindicatur. (La acción de injurias es introducida o por la ley o por la costumbre o por derecho mixto. La ley de las XII Tablas respecto de los versos infamantes, los miembros rotos o los huesos fracturados. Por las costumbres, cuando es realizada cualificadamente se estima por el juez a su arbitrio, y se castiga con una pena congruente con el suplicio).

3 Poena autem iniuriarum ex lege duodecim tabularum propter membrum quidem ruptum talio erat: propter os vero fractum nummariae poenae erant constitutae quasi in magna veterum paupertate. Sed postea praetores permittebant ipsis, qui iniuriam passi sunt, eam aestimare, ut iudex vel tanti condemnet, quanti iniuriam passus aestimaverit, vel minoris, prout ei visum fuerit. Sed poena quidem iniuriae, quae ex lege duodecim tabularum introducta est, in desuetudinem abiit: quam autem praetores introduxerunt, quae etiam honoraria appellatur, in iudiciis frequentatur. Nam secundum gradum dignitatis vitaeque honestatem crescit aut minuitur aestimatio iniuriae: qui gradus condemnationis et in servili persona non immerito servatur, ut aliud in servo actore, aliud in medii actus homine, aliud in vilissimo vel compedito constituatur... (Pero la pena de las injurias según las XII Tablas por un miembro ciertamente roto era el talión: en cambio, por un hueso fracturado las penas dinerarias estaban establecidas en proporción de la gran pobreza de los antiguos. Pero después los pretores permitían a los mismos, quienes han padecido injurias, estimarlas, de modo que el juez condene a tanto, cuanto hubiera estimado la injuria padecida, o a menos, según fuera
De parte de las fuentes extrajurídicas, por el otro lado, resulta de singular importancia el famoso debate entre el filósofo Favorino y el jurista Sexto Cecilio Africano -cuya datación aproximada, de haber ocurrido el diálogo, es el año 146 d. C. $-^{4}$, donde el primero alude, en abono a sus críticas sobre el derecho arcaico, a aquellas que ya hacía Labeón en su obra perdida sobre la Ley de las XII Tablas, según reporta Aulo Gelio, Noctes Atticae 20.1.12-16:

Quod vero dixi videri quaedam esse
inpendio molliora, nonne tibi quoque
videtur nimis esse dilutum, quod ita
de iniuria poenienda scriptum est: "Si
iniuriam alteri faxit, viginti quinque
aeris poenae sunto." Quis enim erittam
inops, quem ab iniuriae faciendae libidine
viginti quinque asses deterreant? 13.
Itaque cum eam legem Labeo quoque
vester in libris quos ad duodecim tabulas
conscripsit, non probaret: "..." inquit "L.
Veratius fuit egregie homo inprobus atque
inmani vecordia. Is pro delectamento
habebat os hominis liberi manus suae
palma verberare. Eum servus sequebatur
ferens crumenam plenam assium; ut
quemque depalmaverat, numerari statim
secundum duodecim tabulas quinque
et viginti asses iubebat." Propterea"

el parecer de este. Pero ciertamente la pena de la injuria, la cual es introducida por la ley de las XII Tablas, cayó en desuso: pero la que introdujeron los pretores, que también se llama honoraria, es frecuente en los juicios. Pues según el grado de dignidad y de vida honesta crece o disminuye la estimación de la injuria: el cual grado de condenación no inapropiadamente también se sigue en la persona esclava, pues una cosa se sanciona para el esclavo representante general, otra para el esclavo de oficio medio, otra para el vilísimo o encadenado...).

4 Véase Casavola, F. $\left(2011^{2}\right)$ : Giuristi adrianei (Roma, "L'Erma" di Bretschneider) pp. 68 y 69; DiliBERTO, O. (1992): Materiali per la palingenesi delle XII Tavole, vol. I (Cagliari, Edizioni AV) p. 159 n. 521. 
inquit "praetores postea hanc abolescere et relinqui censuerunt iniuriisque aestumandis recuperatores se daturos edixerunt. 14. Nonnulla autem in istis legibus ne consistere quidem, sicuti dixi, visa sunt, velut illa lex talionis, cuius verba, nisi memoria me fallit, haec sunt: "si membrum rupit, ni cum e pacto, talio esto." 15. Praeter enim ulciscendi acerbitatem ne procedere quoque exsecutio iustae talionis potest. Nam cui membrum ab alio ruptum est, si ipsi itidem rumpere per talionem velit, quaero, an efficere possit rumpendi pariter membri aequilibrium? In qua re primum ea difficultas est inexplicabilis. 16. Quid si membrum" inquit "alteri inprudens ruperit? quod enim per inprudentiam factum est, retaliari per inprudentiam debet. Ictus quippe fortuitus et consultus non cadunt sub eiusdem talionis similitudinem. Quonam igitur modo inprudentem poterit imitari, qui in exsequenda talione non licentiae ius habet, sed inprudentiae?

\section{Traducción}

Puesto que dije que en realidad pareciera ser ciertamente más leve el impendio ¿no te parece también a ti estar excesivamente diluido? ya que, sobre la injuria, ha sido escrito así: 'Si hizo injuria a otro, sean veinticinco monedas de bronce de pena'. ¿Quién, ciertamente, será tan pobre que veinticinco ases le atemoricen de hacer injurias por placer? 13. Y así, vuestro Labeón tampoco lo aprobó cuando escribió sobre aquella ley en los libros sobre las XII Tablas: 'Ciertamente', inquiere, 'L. Veracio fue un hombre conocidamente ímprobo y de gran insensatez. Él tenía como deleite golpear con la palma de su mano la cara a los hombres libres. Un esclavo lo seguía portando una bolsa llena de ases; de modo que a cada uno que abofeteaba, ordenaba ser pagados los veinticinco ases inmediatamente según la Ley de las XII Tablas'. 'Por ello', inquiere, 'los pretores, luego, establecieron por edicto la abolición de esta y se dejó, según consideraron, la estimación de las injurias a los recuperadores nombrados por ellos'. 14. Pero muchas otras cosas en estas leyes ciertamente, como dije, parecen no poderse aplicar, como aquella de la ley del talión, cuyas palabras, si la memoria no me falla, son estas: 'si ha roto un miembro, y no hay pacto con aquel, haya talión'. 15. Aparte de la crueldad para vengarse, tampoco puede proceder una ejecución justa del talión. Pues, aquel cuyo miembro es roto por otro, si quiere romper igualmente el mismo por talión, pregunto ‘acaso puede lograr el equilibrio en la rotura igualitaria de miembro? Cuestión en que, por principio, esa dificultad es irresoluble. 16. '¿Qué ocurre si rompió el miembro', inquiere, 'a otro imprudentemente? Puesto que ciertamente ha sido hecho por imprudencia, el talión debe ser por imprudencia. El golpe efectivamente fortuito y el meditado no caen bajo un mismo tipo de talión. ¿De qué modo, por tanto, el imprudente podrá ser imitado, por aquel que en ejecución del talión tiene derecho no de licencia, sino de imprudencia?

A los argumentos de Favorino, sigue la réplica de Sexto Cecilio, en Noctes Atticae 20.1.31-38:

Sed enim ipsum vide. Iniurias factas quinque et viginti assibus sanxerunt. Non omnino omnes, mi Favorine, iniurias aere isto pauco diluerunt, tametsi haec ipsa paucitas assium grave pondus aeris fuit; nam librariis assibus in ea tempestate populus usus est. 32. Sed iniurias 
atrociores, ut de osse fracto, non liberis modo, verum etiam servis factas inpensiore damno vindicaverunt, quibusdam autem iniuriis talionem quoque adposuerunt. 33. Quam quidem tu talionem, vir optime, iniquius paulo insectatus es ac ne consistere quidem dixisti lepida quadam sollertia verborum, quoniam talioni par non sit talio neque rumpi membrum facile possit ad alterius rupturae, ut ais tu, "aequilibrium". 34. Verum est, mi Favorine, talionem parissimam fieri difficillime. Sed decemviri minuere atque exstinguere volentes huiuscemodi violentiam pulsandi atque laedendi talione, eo quoque metu coercendos esse homines putaverunt neque eius, qui membrum alteri rupisset et pacisci tamen de talione redimenda nollet, tantam esse habendam rationem arbitrati sunt, ut, an prudens inprudensne rupisset, spectandum putarent aut talionem in eo vel ad amussim aequiperarent vel in librili perpenderent; sed potius eundem animum eundemque impetum in eadem parte corporis rumpenda, non eundem quoque casum exigi voluerunt, quoniam modus voluntatis praestari posset, casus ictus non posset. 35. Quod si ita est, ut dico et ut ipse aequitatis habitus demonstrat, taliones illae tuae reciprocae argutiores profecto quam veriores fuerunt. 36. Sed quoniam acerbum quoque esse hoc genus poenae putas, vae, obsecro te, ista acerbitas est, si idem fiat in te, quod tute in alio feceris? praesertim cum habeas facultatem paciscendi et non necesse sit pati talionem, nisi eam tu elegeris. 37. Quod edictum autem praetorum de aestimandis iniuriis probabilius esse existimas nolo hoc ignores hanc quoque ipsam talionem ad aestimationem iudicis redigi necessario solitam. 38. Nam si reus, qui depecisci noluerat, iudici talionem imperanti non parebat, aestimata lite iudex hominem pecuniae damnabat, atque ita, si reo et pactio gravis et acerba talio visa fuerat, severitas legis ad pecuniae multam redibat.

\section{Traducción}

Pero observa en consecuencia lo mismo. Sancionaron con 25 ases las injurias hechas. Mi Favorino, no rebajaron a tan poco bronce todas las injurias, si bien esta misma pequeña cantidad de ases tuvo el peso de un libra de bronce; pues en aquel tiempo el pueblo tenía en uso el as libral. 32. Sin embargo, en cambio, para las injurias atroces, como respecto del hueso fracturado, no solo las hechas a los libres, sino también a los esclavos, vindicaron por un desembolso por el daño, y para las demás injurias también establecieron el talión. 33. En cuanto ciertamente tú al talión, óptimo varón, has criticado un poco inicuamente y por ello dijiste con cierta graciosa habilidad de palabra no realizarse porque un talión no es par a otro talión ni puede fácilmente romperse un miembro por la rotura de otro, como tú afirmas, con "equilibrio". 34. Es verdad, mi Favorino, que es muy difícil que sea hecho un talión igualísimo. Pero los decenviros, queriendo con el talión disminuir y extinguir tal violencia de golpear y dañar, pensaron que los hombres también son castigados con el miedo a este y creyeron que incluso aquel que había roto un miembro a otro y, sin embargo, no quería paccionar sobre el reemplazo del talión, debía ser arbitrado valorando ser tanta la proporción según si lo rompió prudente o imprudentemente, o en otros términos, el talión, para ello, o lo igualaban a un mismo nivel, o lo pesaban exactamente en librales; pero más bien quisieron que se rompa en la misma parte del cuerpo con el mismo ánimo y con el mismo ímpetu, pero no se exige también la misma circunstancia, 
porque el modo de la voluntad puede ser cumplido, el caso fortuito no puede. 35. De modo que si es así, como digo y como demuestra el mismo hábito de la equidad, aquellos taliones recíprocos tuyos fueron indudablemente más invenciones que verdaderos. 36 . Pero porque también piensas que es cruel este género de penas, ¡oh, te ruego! ¿hay esta crueldad si se hace lo mismo en ti que lo que con seguridad has hecho a otro? especialmente cuando tienes la facultad de paccionar y no hay necesidad de que sea padecido el talión, a menos que tú lo hayas elegido. 37. Pero, puesto que estimas más adecuado el edicto de los pretores sobre la estimación de las injurias, no ignores por ello que según el uso también es reconducido necesariamente el mismo talión a una estimación del juez. 38. Pues si el reo, quien no había querido paccionar, no aceptaba el talión impuesto por el juez, el juez condenaba al hombre por el monto en la estimación del litigio, y así, si por el reo había sido considerado la pacción gravosa y cruel el talión, la severidad de la ley se reducía a una multa de dinero.

Por último, debe añadirse dos testimonios de Festo: uno en que cita al gramático Verrio Flaco para recordar la Tabla 8.25;

\footnotetext{
5 Festo (L. 496), v. Talionis, "Talionis mentionem fieri in XII $(8,2)$ ait Verrius hoc modo, 'si membrum rapsit (rup-), ni cum eo pacit, talio esto'. Neque id quid significet indicat, puto quia notum est; permittit enim lex parem vindictam. (Del talión se hace mención en las XII Tablas $(8,2)$ afirma Verrio que de este modo, 'si rompiera un miembro, y no pacta con aquel, haya talión'. No indica esto lo que signifique, pienso, porque es sabido que la ley ciertamente permite la vindicación a la par). Cfr. Lindsay, M. (1965): "Festus, De verborum significatu”, en W.-M. Lindsay (edit.), en Glossaria Latina, Iussu Academiae Britannicae Edita, IV., Placidus, Festus, J. W. Pirie, W.-M. Lindsay (Hildesheim, Georg OlmsVerlagbuchhandlung) p. 451.
}

y otro, en relación con 8.4 , en que señala la moneda vigente en época decenviral ${ }^{6}$.

A primera vista, el esbozo de las fuentes de transmisión del texto decenviral puede parecer claro. Pero lo cierto es que, en realidad, filológica y dogmáticamente pocas cosas son seguras en la Tabla 8.2,3 y 4 . Debe tenerse en cuenta que ya desde Labeón y hasta Paulo, pasando por Sexto Cecilio Africano y Gayo, viene representada la perspectiva culta de los juristas formados en la historia, la retórica y la gramática, que bajo el signo de la interpretación verbamores, se interesaban en la interpretación de la Ley de las XII Tablas y sus lazos con el derecho actual; esto es, especialmente el del Edicto ${ }^{7}$. De ello, viene que un texto que reconocen recordar por su transmisión oral

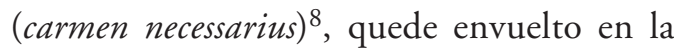
oscura de la fragilidad de los recuerdos, de sus anticipaciones históricas y de sus interpretaciones anacrónicas, como consecuencia de la natural influencia del propio punto de vista de estos observadores ${ }^{9}$. Esto quedará claro, a continuación, con la información que entregaremos en materia de transmisión textual de los preceptos decenvirales.

Como se sabe, a pesar de la aparente concisión con que aparecen reconstruidos estos preceptos, ha habido muchas ediciones diversas debido a los problemas generales que reseñamos a continuación.

\footnotetext{
6 Festo (L. 508), v. Viginti quinque poenae: "Viginti quinque poenae' in XII significat viginti quinque asses ('Veinticinco de pena' en las XII Tablas significa veinticinco ases). Cfr. Lindsay (1965) p. 461.

7 Véase Casavola $\left(2011^{2}\right)$ pp. 1 a 121 (esp. pp. 14 a 16); Diliberto (1992) pp. 179 a 197.

8 Cicerón, De legibus 2.23.59.

9 Pugliese (1940) pp. 4 ss.; Manfredini (1977) p. 21; Pugliese (1978) p. 194; Cursi (2002) p. 222 y n. 3 .
} 
1. Un primer problema textual es la ubicación precisa de este conjunto de normas al interior de las XII Tablas. El antecedente de las reconstrucciones modernas $^{10}$ es la obra del humanista Jacques Godefroy, "Fragmenta XII. Tabularum"11 (1616); más tarde reproducida con leves alteraciones en su obra póstuma "Fontes quattuor iuris civilis"12 (1653). La contribución de Godefroy, merced a su erudita obra, consistió en dotar a la doctrina, desde sus tiempos hasta hoy ${ }^{13}$, de una metodología consistente en suponer que los 6 libros de Gayo sobre comentarios a la Ley de las XII Tablas $^{14}$ sirven como modelo ordenador para la sistematización de las materias de la ley decenviral. Sin embargo, él, a diferencia de las versiones actuales, emplazó los delitos

10 Véase, por todos, con una relación de las diversa ediciones, Ferrary, J.-L. (2005): "Saggio di storia della palingenesi delle Dodici Tavole", en Le Dodici Tavole. Dai Decemviria gli Umanisti, M. Humbert (a cura di), (Iuss Press, Pavia) pp. 503-556.

11 Gothofredus, J. (1616): Fragmenta XII. tabularum, suis nunc primum tabulis restituta probationibus, notis, \& indice munita (Heidelberg, Typis Johannis Lancelloti) pp. 97 a 102

12 Gothofredus, J. (1653): Fontes quattuor iuris civilis in unum collecti (Legis XII. Tabularum Fragmenta...), (Gevenae, Sumptib. Ioannis Ant. \& Samuelis de Tournes) pp. 112 a 124 (esp. pp. 117, 121 y 123).

13 Véase, con síntesis de la doctrina y proporcionando nuevos puntos de vista, Lenel, O. (1892): Das Sabinussystem (Strassburg, Verlag von Karl J. Trübner) pp. 6 ss. (= (1990): Gesammelte Schriften, II (Napoli, Jovene Editore); Lauria, M. (19632): Ius Romanum, I.1. (Napoli, L’Arte Tipografica) pp. 50 ss.; WIEACKER, F. (1965): "Rc. M. Lauria, Ius Romanum I, 1" Iura 16, pp. 269-283; Schiller, A. A. (1978): Roman Law: Mechanisms of Development (The HagueParis-New York, Walter de Gruyter ) pp. 395 a 397; Amirante, L. $\left(1991^{2}\right)$ : Studi di storia costituzionale romana (Napoli, Jovene Editore) p. 133 a 148; DilIBERTO (1992) pp. 9 a 21.

14 Cfr. Lenel, O. (2000): Palingenesia iuris civilis, I (Roma, Il Cigno Galileo Galilei, pero, Leipzig, B. Tauchnitz,1889) cols. 242 a 246. en la Tabla VII (De Delictis) ${ }^{15}$, la cual presentaba -según su conjetura- una tercera ley referida a las injurias (Lex de iniuriis) ${ }^{16}$, la que a su vez se subdividía en un primer capítulo sobre las injurias leves (De iniuriis levioribus) ${ }^{17}$, un tercer capítulo sobre el miembro roto (De membro rupto $)^{18}$ y un cuarto sobre el hueso fracturado (De osse fracto $)^{19}$. En suma, para Godefroy, la ubicación de nuestra materia en la Ley de las XII Tablas sería la siguiente: VII.3.1 $(=8.4)$; VII.3.3 (=8.2); y, VII.3.4 (=8.3).

El actual emplazamiento de la materia (Tabla 8) y el orden interno de la secuencia de preceptos (2,3 y 4 ), solo aparecieron dos siglos después, con la edición de Dirksen $(1824)^{20}$; la cual se transformó en el canon -o, al menos, el modelo ampliamente dominante en este punto- ${ }^{21}$, merced a su recepción en las posteriores ediciones - por nombrar las más relevantes-, de Bruns $(1860)^{22}$, Schoell $(1866)^{23}$, Mommsen

\footnotetext{
15 Fragmenta XII. tabularum, p. 93; Fontes quattuor, p. 112.

16 Fragmenta XII. tabularum, p. 97; Fontes quattuor, p. 116.

17 Fragmenta XII. tabularum, p. 97; Fontes quattuor, p. 117.

18 Fragmenta XII. tabularum, p. 100; Fontes quattuor, p. 121.

19 Fragmenta XII. tabularum, p. 102; Fontes quattuor, p. 123.

20 Dirksen, H. E. (1824): Uebersicht der bisherigen Bersuche zur Kritik und Herstellung des Textes der Zwölf-Tafel-Fragmente (Leipzig, J. C. Hinrichsche Buchhandlung) pp. 516 a 529.

21 En términos generales, acaso deba considerarse más exitosa la palingenesia ofrecida por Schoell, en 1866, de acuerdo, en cualquier caso, a las líneas maestras trazadas por Dirksen, como señala AMIRANTE $\left(1991^{2}\right)$ p. 134

22 Bruns, G. (1860): FIRA (Tübingen, Laupp\&Siebeck) p. 11

23 Schoell, R.(1866): Legis Duodecim Tabularum Reliquiae (Leipzig, B. G. Teubner) pp. 141 y 142.
} 
$(1887)^{24}$, Bonfante $(1903)^{25}$, Gradenwitz $(1909)^{26}$, Riccobono (1941) 27 y GirardSenn $(1967)^{28}$.

No obstante, esta sedes materiae no puede considerarse pacífica. En el siglo XIX, la edición de Voigt (1883) ${ }^{29}$ volvió a ubicar las disposiciones en la Tabla VII, como ya estaba en Godefroy; pero, a diferencia de este, en el mismo orden interno de la secuencia moderna de los preceptos; esto es: VII.14 (=8.2), VII.15 (=8.3) y VII.16 (=8.4). Por su parte, en el siglo XX, Amirante $^{30} \mathrm{y}$, especialmente, Diliberto ${ }^{31}$, propusieron sustituir el modelo ordenador -los comentarios de Gayo a la Ley de las XII Tablas-, por la estructura de la correspondiente narración de las Noctes Atticae, de Aulo Gelio (avanzada la primera mitad del siglo II d. C.); donde las injurias aparecen tratadas al hilo de la in ius vocatio. La idea fue recogida, en efecto, en la edición de Crawford (1996) ${ }^{32}$, quien, habida cuenta de que la in ius vocatio es una de las escasas materias que sabemos con gran seguri-

24 Bruns, G.; Mommsen, Th. $\left(1887^{5}\right)$ : FIRA (Tübingen, P. Siebeck) p. 28.

25 Bonfante, P. (19233): Storia del Diritto Romano, 1 (Milano, Società editrice Libraria, pero, 1903) p. 204.

26 Bruns, G.; Mommsen, Th.; Gradenwitz, O. (19097): FIRA, Leges et Negotia (Tübingen) p. 29.

27 Riccobono, S. (1968): FIRA, Leges (Firenze, S. A. G. Barbèra, pero, 1941) pp. 53 y 54.

28 Girard, P.; SenN, F. et alii (1977): Le lois des Romains, 7édition par un groupe de romanistes des "Textes de droit romain", Tome II, V. Giuffrè (a cura di), (Napoli, Jovene Editore, pero, 1967) p. 63.

29 Voigt (1883) p. 722.

30 Amirante, L. (1992): "Un'ipotesi di lavoro: le 'sequenze' e l'ordine delle norme decemvirali", Index 20, pp. 205-210.

31 Diliberto, O. (1992): "Contributo alla palingenesi delle XII Tavole. Le 'sequenze' nei testi gelliani”, Index 20, pp. 229-277; y, especialmente, Diliberto (1992) "Materiali...".

32 Crawford, M. H. (1996): Roman Statutes, vol. II (London, University of London) p. 606. dad que se encontraba en la Tabla I -gracias a Cicerón, de legibus 2.4.9 y 2.23.59_33, ofreció la misma secuencia de los preceptos que se hallaba en las anteriores ediciones, pero ahora precisamente en la Tabla I.13 $(=8.2)$, I.14 (=8.3) y I.15 (=8.4).

2. Un segundo problema, corresponde a las lecciones de cada uno de los preceptos de la Tabla 8.2,3 y 4, individualmente considerados. A partir de las distintas fuentes que sirven para reconstruir el texto decenviral, las lecciones asumidas por los editores dependen en buena medida de cuál de ellas estos hayan escogido como modelo principal y cuál de cada una de sus transmisiones textuales y ediciones críticas haya sido, a su vez, la preferida. Por supuesto, aquí no podemos abordar todo esto en detalle, por lo cual nos remitimos a los aparatos críticos de las ediciones citadas. Solo haremos alusión a aquello que, a nuestro modo de ver, tiene importancia según nuestro actual propósito.

La Tabla $8.2^{34}$ se presenta, en cuanto ahora interesa, con un contenido relativa-

33 Véase, por todos, Lauria $\left(1963^{2}\right)$ p. 26; DiliBerto (1992) p. 108; Ferrary (2005) p. 535; DiliBERTo, O. (2005): “Una palingenesi 'aperta", en Le Dodici Tavole. Dai Decemviri agli Umanisti, M. Humbert (a cura di), (Pavia, Iuss Press) pp. 222 y 223.

34 Godefroy: Fragmenta XII. tabularum, 7.3.3. SI MEMBRVM RVPSIT, NI CVM EO PACIT, TALIO ESTO (p. 100) = Fontes quattuor (p. 121); DIRKSEN: 8.2. SI MEMBRVM RVPIT, NI CVM EO PACIT, TALIO ESTO (p. 516); lo sigue Bruns: 8.2. si membrum rupit, ni cum eo pacit, talio esto (p. 11); vuelve a la formulación de Godefroy, Schoell: 8.2. SI MEMBRVM RVPSIT, NI CVM EO PACIT, TALIO ESTO (p. 141); sigue ahora a Dirksen, Voigt: 7.14: Si membrum rupit, ni cum eo pacit, talio esto (p. 722); una vez más con Godefroy, Mommsen: 8.2. SI MEMBRUM RUP[S]IT, NI CUM EO PACIT, TALIO ESTO (p. 28); Bonfante: 8.2. Si membrum rupsit, ni cum eo pacit, talio esto (p. 204); GradeNwITZ: 8.2. SI MEMBRUM RUP $<$ S $>$ IT, NI CUM 
mente estable que oscila entre los modelos de Godefroy y Dirksen (rupsitlrupit). Sin embargo, se debe proceder con cautela a aceptar esta formulación del texto decenviral, pues Favorino reconoce estar recordando el precepto simplemente de memoria, en Noctes Atticae 20.1.14: "cuius verba, nisi memoria me fallit, haec sunt" (cuyas palabras [sc. de la Tabla 8.2], si la memoria no me falla, son estas).

La Tabla $8.3^{35}$, en cambio, presenta algunas variaciones que dependen de la mayor o menor relevancia de atribuida por

EO PACIT, TALIO ESTO (p. 29); Riccobono: 8.2. SI MEMBRUM RUP[S]IT, NI CUM EO PACIT, TALIO ESTO (p. 53); varía el tiempo verbal, GirARD-SEnN (7a ed.): 8.2. SI MEMBRVM R[V] PS $<<$ ER $>>$ IT, NI CUM EO PA[C]IT, TALIO ESTO (p. 63); por último, nuevamente en la línea de Dirksen, Crawford: 1.13. SI MEMBRUM RUPIT, NI CUM EO PACIT, TALIO ESTO (p. 606).

35 Godefroy: Fragmenta XII. tabularum,7.3.4. QVI OS EX GENETALI FVDIT LIBERO, CCC: SERVO, CL. AERIS POENAE SVNTO (p. 102) = Fontes quattuor (p. 123); Dirksen: 8.3. Propter os uero fractum aut collisum trecentorum assium poena erat (ex lege XII. Tabularum), velut si libero os fractum erat; at si seruo, centum et quinquaginta (p. 521); Bruns: 8.3. Ex lege XII. tab. propter os uero fractum aut collisum CCC assium poena erat, si libero os fractum erat, at si seruo, CL (p. 11); Schoell: 8.3. MANV FVSTIVE SI OS FREGIT LIBERO CCC, SI SERVO CL, POENAM SUBITO [SESTERTIORVM] (p. 141); VoIgT: 7.15. Manu, fusti si os fregit homini libero, CCC, servo, CL poenae sunto (p. 722); MoMmsen: 8.3. MANU FUSTIVE SI OS FREGIT LIBERO, CCC, [SI] SERVO, CL POENAM SUBITO (p. 28); Bonfante: 8.3. Manu fustive si os fregit libero, CCC, si servo, CL poneam subito (p. 204); Gradenwitz: 8.3. MANU FUSTIVE SI OS FREGIT LIBERO, CCC, SI SERVO, CL POENAM SUBITO. (p. 29); RicCOBono: 'manu fustive si os fregit libero, CCC, (si) seruo, CL poenam subit sestertiorum'. (p. 53); GIRARD-SENN (7a ed.): MANV FVSTIVE SI $<<$ MANIFEST $>>$ OS FREGIT $<$ COLLISETVE $>$ LIBERO CCC, (SI) SERVO, CL POENA $<<\mathrm{M}>><$ SVNTO $>$ SVBIT $<<\mathrm{O}>>$ SE[S]TERTIORUM (p. 63); CRAWFord: 1.14. si os fregit libero, CCC, $<$ si $>$ seruo, CL poena $<$ e $>$ su $<$ n $>$ to $($ p. 606). los editores al texto de Gayo, Inst. 3.223 y, especialmente, a las variadas ediciones y tradiciones textuales de Paulo, Collatio 2.5.5.

Nosotros, al inicio, hemos preferido la lección "si os fregit libero, CCC, si servo, CL poenam subito", no aquella que señala "manu fustive si os fregit...". Esta segunda opción, sin embargo, es la preferida en la mayoría en las ediciones de la Ley de las XII Tablas -esto es, a excepción de los editores hasta Bruns, inclusive, $\mathrm{y}$, hoy, de Crawford-. La razón para contemplar el "manu fustive" está en los códices que contienen la Collatio $^{36}$. Es cierto que el códice Berolensis (descubierto en el siglo XVI), comienza la cita de la Tabla 8.3, directa y secamente, diciendo "uelut illa: si os fregit libero, CCC si seruo, CL poenam subito sestertiorum" 37 ; lo cual parece dar con el texto decenviral -tal como a nosotros nos parece lo más probable-, a partir del cotejo con Gayo, Inst. 3.223: "propter os uero fractum aut conlisum trecentorum assium poena erat, si libero os fractum erat; at si seruo, CL". Sin embargo, en el siglo XIX aparecieron los códices Vercellensis, que antepone "Manifestos", y Vindobonensis, que señala "Manifestus". Observados estos por Lachmann ${ }^{38}$, este corrigió y sustituyó di-

\footnotetext{
36 Como se sabe, los tres códices fundamentales que contienen la Collatio son el Berolensis Lat. fol. 269 , del siglo IX, y los códices Vercellensisn. 122 y Vindobinensisn. 2160, ambos del siglo X. Mientras el primero ya fue manejado por los juristas del siglo XVI, los dos restantes fueron descubiertos, de manera independiente, apenas en 1822. Véase Mommsen, Th. (1890): "Mosaicarum et Romanarum Legum Collatio", en Collectio Librorum Iuris Anteiustiniani in Usum Scholarum, 3, P. Krueger; Th. Mommsen; G. Studemund, apud (Berolini,Weidmannos) pp. 109 a 112.

37 Así aparece todavía en Baviera, J. (1968): FIRA, 2, Auctores (Firenze, S. A. G. Barbèra, pero, 1940) p. 550. 38 Lachmann, K. (1840): "Verbesserungen des Textes der Collatio", Zeitschrift für geschichtliche Rechtswissenschaft 10 (1840) 2 pp. 309-314 (esp. pp.
} 
chas expresiones por "Manu fustive" 39 , quedando así la lección de la Tabla 8.3 como "manu fustive si os fregit...". Lachmann ${ }^{40}$ respaldó su proposición en el contexto ofrecido por Gayo, Inst. 3.220 ("pugno puta aut fuste percussus"); lo cual también puede reforzarse, como destaca Riccobono ${ }^{41}$, con Paulo, Collatio 2.6.4 ("pugno puta an fuste an lapide sicut formula proposita est"), Gayo, D. 50.16 .41 ("Armorum appellatio -significat- et fustes et lapides") $\mathrm{y}$, entre las fuentes literarias, Plauto, Asinaria 2.2.104 ("pugno malam si tibi percussero"). Sin embargo, es claro, como veremos, que las XII Tablas aparecen concentradas en delitos de resultado -en este caso, fracturar un hueso-, no de actividad; por lo tanto, que el hueso se fracture con la mano o con el bastón parece más propio de la posterior contextura edictal del delito de injurias -en cuya fórmula debía indicarse la conducta concreta que causaba injuria- ${ }^{42}$. De hecho, el texto de Plauto, Asinaria 2.2.104 sirvió a Lenel ${ }^{43}$ precisamente para estructurar la

310 y 311) (= (1876): Kleinere Schriften, 2, G. Reimer, Berlin, p. 242).

39 La corrección también es aceptada por Huschke, pero prescindiendo de la partícula "ve"; por lo que resulta "manu fusti". Cfr. Huschke, E. (18865): Iurisprudentia Anteiustinianae quae supersunt (Lipsiae, B. G. Teubneri in aedibus) p. 564 y n. 5. Esta es la lección aceptada por Voigt para la que él supone ser la Tabla VII.15. Cfr. Voigt (1883) p. 722

40 Lachmann (1840) p. 311 (= (1876) p. 242).

41 Cfr. Riccobono (1941) p. 54 n. 3.B.

42 Ulpiano, 57 ad Ed., D. 47.10 .7 pr. Véase, por todos, Lenel, O. $\left(1927^{3}\right)$ : Das edictum perpetuum. Ein Versuch zu seiner Wiederherstellung (Leipzig, Verlag von Bernhard Tauchnitz) p. 398.

43 Junto a GaYo, Inst. 4.60 (“...aut si is, cui pugno mala percussa est, in actione iniuriarum etiam aliam partem corporis percussam sibi demonstraverit..."), SÉNECA, De beneficiis 2.35.2 ("qui illum pugno percusserit, iniuriarum damnabitur") y Suetonio, Vita Vitelii c. 7 (“... iniurirarum formulam, quasi calce ab eo percussus, intendisse...”). Véase Lenel (192733) p. 398 n. 7. fórmula -demonstratio- que hipotéticamente correspondió en época clásica al delito de injurias -si bien se discute su importancia para determinar la datación del edicto pretorio en esta materia-; asimismo, el discurso de Gayo, Inst. 3.220, se refiere también al delito edictal, en ningún caso al delito decenviral (Inst. 3.223); y Paulo, Collatio 2.6.4, señala expresamente "sicut formula proposita est". Por todo ello, los textos de la Collatio 2.5.5 según los códices Vercellensis y Vindobonensis -que por lo demás, no dicen "manu fustive", sino que esto es una interpretación-, no parecen reproducir fielmente el texto decenviral.

Pero, como sea, ya desde Momm$\operatorname{sen}^{44}$ parece que se debe aceptar que ninguna de estas fuentes, ni Paulo, Collatio 2.5.5, ni Gayo, Inst. 3.223, contienen el texto preciso de la Tabla 8.3, sino una alusión más o menos libre. Basta pensar que en Gayo, Inst. 3.223, no solo se habla de os fractum, sino también conlisum -razón por la cual la edición de Girard-Senn ${ }^{45}$ lo adjunta al texto decenviral $(<>)$, "fregit <collisetve $>$ ", a pesar de que ello no es procedente ya que esto no parece haber formado parte de la obra original, sino que es un glosema ${ }^{46}$. O bien, que la pena de 300 y 150, corresponde a "ases" según Gayo, Inst. 3.223, y a "sestercios" según la Collatio 2.5.5 ("CCC... CL... sestertiorum”). Por cierto, los sester-

44 Bruns; Mommsen (18875) p. 28 n. 3.4.

45 Girard; SENn et alii (1977) p. 63.

46 El término "conlisum” en Gayo, Inst. 3.223 parece ser un glosema, por la falta de correspondencia dentro del texto: primero dice "propter os uero fractum aut conlisum" y, después, "si libero os fractum erat". Véase Di Paola, S. (1946-7): "La genesi storica del delitto di iniuria", en Annali del Seminario Giuridico dell'Università di Catania 1, p. 268 n. 2; SolazzI, S. (1971): "Appunti di critica gaiana", en Scritti di Diritto Romano, V (1947-1956), (Napoli, Casa Editrice Dott. Eugenio Jovene) p. 459 n. 63. 
cios indudablemente son un anacronismo, y deben ser descartados. Pero los ases también presentan dificultades. Es claro que en época clásica las sanciones de las respectivas prescripciones de 8.3 y 8.4 se recordaban en ases, salvo en la Collatio; para la Tabla 8.4 , Gayo, Inst. 3.223, señala " $X X V$ assium", y Gelio, Noctes Atticae 20.1.12, "viginti quinque aeris"; lo cual se confirma con el testimonio de Festo (L. 508) "Viginti quinque poenae' in XII significat viginti quinque asses ('Veinticinco de pena' en las XII Tablas significa veinticinco ases $)^{47}$. Es más, es mérito de Diliberto ${ }^{48}$ haber observado que Gelio da dos versiones de la pena de la Tabla 8.4; en las Noctes Atticae 20.1.12 dice "vinginti quinque aeris" -como hemos recordado-, mas en 10.6 .8 señala "viginti quinque asses", lo que podría demostrar que el autor contó para la redacción de su obra con dos versiones de la Ley de las XII Tablas -aquella que se refería a los ases era más antigua-, ninguna de las cuales era completamente fiel al original -que en este punto parece corresponder a la versión de Festo-. Tal como ya expresaba Solazzi ${ }^{49}$, parece extraño que en la Tabla 8.3 y 4 , se hable de ases cuando solo un siglo más tarde comenzó la primera acuñación de monedas de bronce. En efecto, Sexto Cecilio Africano, según las Noctes Atticae 20.1.31 habría señalado: "...tametsi haec ipsa paucitas assium grave pondus aeris fuit; nam librariis assibus in ea tempestate populus usus est" (si bien esta misma pequeña cantidad de ases tuvo el peso de un libra de bronce; pues en aquel tiempo el pueblo tenía en uso el as libral).

\footnotetext{
47 Cfr. Lindsay (1965) p. 461.

48 Diliberto, “Materiali...” (1992) pp. 212 y 213.

49 Solazzi, S. (1955): "La questione dell'autenticità delle Dodici Tavole", en Scritti di Diritto Romano, I (1889-1913), (Napoli, Casa Editrice Dott. Eugenio Jovene) p. 87.
}

En realidad, como señala De Martino ${ }^{50}$ a la época de la promulgación de las XII Tablas, no había ases librales ni sestercios, como dicen estas fuentes tardías, sino que el bronce era pesado. Algo similar señalaron después Manfredini ${ }^{51}$ y Pugliese $^{52}$, para quienes el texto decenviral seguramente fijaba las penas en aes signatum o aes rude $\mathrm{O}$, derechamente, en animales ${ }^{53}$. Y, en efecto, esto es con bastante verosimilitud lo que debió haber aparecido realmente en la Ley. Seguramente estas dudas llevaron a que Schoell cancelara la mención "aeris" del texto decenviral de la Tabla 8.4 reportado por Gelio, Noctes Atticae 20.1.12 ("viginti quinque aeris poenae sunto"); ello, en vista de que ya Festo no señala ninguna determinación para "25" (“Viginti quinque poenae"). Albanese $^{54}$ no le atribuye importancia a la cancelación, aunque da por sentado que en época decenviral, según el propio Gayo $1.122^{55}$, el dinero se pesaba y por tanto

\footnotetext{
50 De Martino, F. $\left(1973^{2}\right)$ : Storia della costituzione romana, 2 (Napoli, Casa Editrice Dott. Eugenio Jovene) p. 8 n. 17.

51 Manfredini (1977) pp. 68 a 70

52 Pugliese (1978) p. 195.

53 Tiene razón Cursi cuando, siguiendo a Pugliese (n. anterior), señala que el hecho de que se pesara el bronce no implica la inaplicabilidad de estas normas por la falta de dinero de curso legal; pues el aes signatum o rude, pesado por un libripens, habría sido suficiente para medir el valor de los bienes. Además, en lo que hace al pago con animales, ya las leges Aternia Tarpeia (454 a. C.), Menenia Sextia (452 a. C.) e Iulia Papiria (430 a. C.), establecieron una suficientemente detallada correspondencia entre pecudes y asses. Véase Cursi (2002) p. 230 n. 35.

54 Albanese, B. (1980): "Una congetura sul significato di «iniuria» in XII tab. 8.4”, IVRA 31, p. 23.

55 GAYO, Inst. 1.122: "ideo autem aes et libra adhibetur, quia olim aereis tantum nummis utebantur... sicut ex lege XII tabularum intellegere possumus; eorumque nummorum vis et potestas non in numero erat, sed in pondere... Tunc igitur et qui dabat alicui pecuniam, non numerabat eam, sed appendebat; unde servi, quibus permittitur administratio pecuniae, dispensatores
} 
ha intervenido aquí una actualización del monto de la pena. Para nosotros, en cambio, la cuestión tiene mucha importancia porque la lectura que se hace de estos delitos decenvirales tiene siempre en su base la presunta gravedad de los mismos, deducida en correlación con la entidad de sus penas, las cuales, en realidad, son dudosas. En fin, todo esto parece demostrar que las lecciones que se han reconstruido no son seguras.

Finalmente, las ediciones también parecieran bastante estables en lo que hace a la lección de la Tabla $8.4^{56}$, tomada fundamentalmente de la obra de Aulo Gelio, Noctes Atticae 20.1.12, y de la misma Co-

appellati sunt" (Pero por ello se usaba un cobre y una balanza, porque en aquel tiempo se usaban cantidades de monedas de bronce... tal como podemos observar en la Ley de las XII Tablas; y la fuerza y potestad de estas monedas no estaba en el número, sino en el peso... Entonces, por tanto, también quien daba dinero a alguno, no lo contabilizaba, sino que lo pesaba; de donde que los esclavos, a los que se les permite administrar dinero, son llamados dispensadores).

56 Godefroy: Fragmenta XII. tabularum, 7.3.1. SI QVI INIVRIAM ALTERI FAXIT, XXV. AERIS POENAE SVNTO (p. 97); Fontes quattuor, 7.3.1: SI INIVRIAM ALTERI FAXIT, XXV. AERIS POENAE SVNTO (p. 117); DIRKSEN: 8.4. SI INIVRIAM FAXIT ALTERI, VIGINTI QVINQVE AERIS POENAE SUNTO (p. 525); BRUNs: 8.4. Si injuriam faxit alteri, viginti quinque aeris poenae sunto. (p. 11); Schoell: 8.4. SI INIVRIAM [ALTERI] FAXSIT, VIGINTI QVINQVE POENAE SVNTO (p. 142); Voigt: 7.16. Si iniuriam alteri faxit, XXV poenas sunto. Qui occentassint, verberantor (p. 722); MoMmsen: 8.4. SI INIURIAM FAXSIT, VIGINTI QUINQUE POENAE SUNTO (p. 28); BONFANTE: 8.4. Si iniuriam faxsit, viginti quinque poenae sunto (p. 204); GRAdENwITZ: 8.4. SI INIURIAM FAXSIT, VIGINTI QUINQUE POENAE SUNTO. (p. 29); RICCOBONo: 8.4. SI INIURIAM [ALTERI] FAXSIT, VIGINTI QUINQUE POENAE SVNTO (p. 54); Girard-SENN (7a ed.): 8.4. SI INIVRIA $<<$ ALTERI $>>$ FAXSIT, <ALTERI > VIGINTI QVINQVE <<AERIS >> PO[E]NA[E] SVNTO (p. 63); Crawford: 1.15. si iniuriam ?alteri? faxsit, uigintiquinque poenae sunto (p. 606). llatio 2.5.5. Pero el asunto es mucho menos pacífico de lo que aparenta.

Una primera cuestión es el monto de la pena-que acabamos de tratar, y debe ser muy tenido en cuenta-.

La segunda cuestión es la pertinencia del dativo "alteri". Godefroy estableció la lección tradicional, "Si iniuriam alteri faxit", que es la seguida por Voigt. Pero Dirksen y Bruns pusieron en duda su ubicación y lo trasladaron después de faxsit, "Si iniuriam faxsit alteri"; todo lo cual es asumido por Girard-Senn, que indica en signos críticos que alteri debe expulsarse del lugar tradicional $(<<>>)$, y, aunque no aparezca así en el original, adjuntarse al texto (<>) después de faxsit, "Si iniuria <<alteri>> faxsit, <alteri>". Ya antes, Schoell había puesto en duda que alteri debiera aparecer en cualquier lugar, "Si iniuriam [alteri] faxsit", lo cual fue seguido por Riccobono y Crawford. En cambio, Mommsen, Bonfante y Gradenwitz, derechamente suprimieron la palabra, "Si iniuriam faxsit".

A pesar del debate editorial, la lección "Si iniuriam alteri faxit" parece bastante acreditada. Fraenkel ${ }^{57}$ critica la expulsión de la palabra -en lo cual es seguido por Simon ${ }^{58}$, Birks $^{59}$, Halpin ${ }^{60}$, Albanese ${ }^{61}$ y

\footnotetext{
57 Fraenkel, E. (1925): "Franz Beckmann, Zauberei und Recht in Roms Frühzeit. Ein Beitrag zur Geschichte und Interpretation des Zwölftafelrechtes, Diss. Münster 1923. 71 S. [Druck von W. Nolte, Osnabrück]", Gnomon. Kritische Zeitschrift für die gesamte klassische Altertumswissenschaft 1 (1925) 4 p. 191 y n. 1. (= (1964): "Anzeige von Beckmann, Zauberei und Recht in Roms Frühzeit", en Kleine Beiträge zur klassische Philologie, 2. Zur römischen Literatur zu juristischen Texten verschiedenes (Roma, Edizioni di Storia e Letteratura) p. 404 y n. 1.

58 Simon (1965) p. 134

59 Birks (1969) p. 189.

60 Halpin (1976) p. 346.

61 Albanese (1980) pp. 23 y 24.
} 
Cursi $^{62}-$, por cuanto la lección con alteri aparece atestiguada tanto en las Noctes Atticae 20.1.12 como en la Collatio 2.5.5 -lo cual es, en nuestra opinión, lo más decisivo-, y porque la estructura de la lección se muestra como una construcción perfectamente posible en materia jurídica; en cuanto coincide con la estructura del tercer capítulo de la lex Aquilia de damno, "si quis damnum alteri faxit"63 -a lo cual volveremos-, al igual que con el testimonio ciceroniano ${ }^{64}$ de otras normas decenvirales, "quod infamiam faceret flagitiumve alteri" $(8.1)^{65}$, y con la lex Osca tabulae Bantinae 3 (11. 13-14), "Siquis pro magistratu alteri fundi aut pecuniae diem dixerit" (= "svaepis pru meddixud altrei castrovs avti eituas zicolom dicust") y 5 (11. 23-24), "siquis apud eos cum altero lege agere volet" (="svaepis op eizois com atrud ligud acum herest").

La tercera cuestión relativa a la Tabla 8.4, se refiere a la lección de las Noctes Atticae 20.1.12 "iniuriam-faxsit" ("hace-injuria", con el acusativo "iniuriam"), la cual se debe a la intervención editorial de los modernos filólogos; pues la realidad es, en cambio, que los códices de las Noctes Atticae recogen "iniuria-faxsit" ("injustamentehace", con el ablativo adverbial "iniuria"). Si ello ha sido así, es porque los filólogos se han dejado influir por los juristas editores de la Ley de las XII Tablas, como veremos a continuación.

La famosa edición de Hosius, señala para Noctes Atticae 20.1.12 (l.14): "(VIII

\footnotetext{
62 Cursi (2002) pp. 232.

63 Ulpiano, 18 ad Ed., D. 9.2.27.5.

64 Cicerón, De re publica. 4.10.12.

65 Que esta norma no parece tener vínculo originalmente con el delito de injuria, también es dicho por Fraenkel (1925) p. 197 (=(1964) p. 412).

66 Cfr. Riccobono (1941) p. 164.
}

4): Si iniuriam alteri faxsit" 67 . Es decir, se inclina por el acusativo "iniuriam". Pero en el aparato crítico señala "14. iniuria $\omega$ "68; es decir, que hay consenso en los códices en cuanto al ablativo adverbial "iniuria" 69 Pero, entonces ¿por qué Hosius no prefirió esta última declinación? La razón ya está preanunciada en la cita del texto, pues la inserción de la ubicación de la norma en la Ley de las XII Tablas, "(VIII 4)", denota que ha seguido a los editores de esta. Esta primera impresión, queda luego confirmada por la declaración expresa de Hosius, quien en una nota a la línea 14, señala: "14 $A d$ singulas leges $v d$. Bruns"70. En todo caso, al seguir las ediciones jurídicas, Hosius no ha hecho más que continuar la tradición de los filólogos. Ya Hertz, su antecesor, en la edición teubneriana de 1871 , entregaba el texto de 20.1.12 restituido con ciertas dudas para lograr el acusativo: "Si iniuria $[m]$ alteri faxsit" 71 . Y, posteriormente, en la edición de 1883, el mismo Hertz señalaba para 20.1 .12 (l. 53): "Si iniuriam alteri faxsit", sin signos críticos; pero, a cambio, anotaba dentro del aparato crítico que ahora ofrecía:

\footnotetext{
67 Hosius (ed.), (1903): A. Gellii. Noctium Atticarum Libri XX. Post Martinum Hertz..., 2 (Lipsiae, B. G. Teubneri in aedibus) p. 287.

68 El signo " $\omega$ " significa "consensus codicum aut omnium aut reliquorum" (consenso de los códices o de todos o de los restantes). Cfr. Hosius (1903) p. LXIV.

69 Véase, con una explicación sintética de los códices de las Noctes Atticae, Reynolds, L. D. (1983): Texts and transmission. A survey of latin classics (Oxford, Oxford University Press) pp. 176 a180.

70 "Bruns = Fontes Iuris Romani ed. Bruns", cfr. HosIUs (1903) p. LXIV.

71 Hertz, M. (1871): A. Gellii. Noctium Atticarum Libri XX, 1 (Lipsiae, B. G. Teubneri sumptibus et typis) p. 240. Como se sabe, el signo " $s$ " se refiere a los códices más recientes (codd. recentiores). Cfr. Hertz, M. (1883): A. Gellii. Noctium Atticarum Libri $X X, 1$ (Berolini, impensis Wilh. Hertz, Librariae Besserianae) pp. VI y esp. 441.
} 
"leg. XII tabb. VIII 4 p. 525 Dirksen; p. 142 RSchoell; p. 27 CGBruns FIRA ${ }^{5}$; VII 16 t. I p. 722 MVoigt cf. Paul. lib. sing. et tit. de iniuriiscoll. II 5, 5 'qui iniuriam alteri facit, quinque et viginti sestertiorum poenam (poena al.) subit'|| 53 iniuriam] sPaulus l. l.; Ascens. 1536: iniuria (=ऽ)"72. Es decir, Hertz disipó sus dudas sirviéndose, ante todo, de las diversas ediciones jurídicas de la disposición generalmente ubicada en la Tabla 8.4, las cuales cita con exactitud. Además, en cuanto al acusativo “iniuriam”, señala que este ya se encuentra en la edición preparada en 1536 por Aegidius Maserius (Gilles de Maizières) y Petrus Mosellanus, con los escolios de Jodocus Badius Ascensius, "Ascens. 1536", donde en efecto se lee:"Si *iniuriam alteri facit" 73 , pero aparece el escolio del texto indicando "*iniuria" 74 , precisamente en atención a lo atestiguado por los códices; lo cual es reforzado por Hertz, quien añade mediante el signo " $=\varsigma$ "75, que esta, “iniuria" (en ablativo adverbial), es la lección también en los códices más recientes de la obra geliana y también en los códices más recientes que contienen el texto de Paulo, en la Collatio 2.5.5.

Inmediatamente indagaremos sobre la alusión de Hertz a la lección de la propia Collatio, pero antes de atender a ello, nos parece que ha quedado bien ilustrada la

\footnotetext{
72 Hertz (1883) p. 441.

73 Maserius, AE.; Mosellanus, P.; Ascensius, J. B. (1536): Auli Gellii Noctivm Atticarvm Lib. XIX: (Nam octauus praeter capita desideratur) pluribus locis quam ante hac integrioris. Cum Ascensianus scholiis, collectis fere ex annotatis sane doctorum hominum AEgidii Maserii Parisiensis, \& Petri Mosellani Protogensis, in oficina Ioannis Parvi, s./l. (pero, Paris) fol. 142 vlta.

74 Maserius; Mosellanus; Ascensius (1536): fol. 142 vlta.

75 Como se sabe, el signo " $\mathrm{s}$ " se refiere a los códices más recientes (codd. recentiores). Cfr. Hertz (1883) p. VI.
}

especie de círculo vicioso que se ha producido: los juristas se nutren del texto de Aulo Gelio para reconstruir la Ley de las XII Tablas, al turno que los filólogos se sirven del texto de las XII Tablas para reconstruir el de Aulo Gelio.

En lo que respecta a la Collatio, la edición de Mommsen señala para 2.5.5 ( $l$. 9), en efecto: "qui iniuriam alteri facit"76; es decir, aparece la lección con el acusativo "iniuriam". Sin embargo, ya el aparato crítico indica "iniuria V"77; lo cual significa que en el códice Vercellensis, datado en el siglo X, pero descubierto en 1822, también se encuentra el ablativo adverbial "iniuria". La cuestión, entonces, es que desde el siglo XVI el acusativo "iniuriam" del Berolensis había servido de canon único de la Collatio y, como era obvio, pasó a ser la lección tradicional que nutrió tanto las ediciones de esta como las de las Noctes Atticae 20.1.12 y, desde luego, las de la Tabla 8.4. De hecho, el primer comentarista de la Collatio, Petrus Pithoeus -quien tuvo conocimiento del documento hacia $1570{ }^{78}$, señaló "Qui iniuriam] haec sunt verba legis ipsius XII. tab."79, y, confiado en que verdaderamente tenía entre sus manos las palabras precisas de la norma decenviral -entre ellas, por supuesto, "iniuriam"-, procedió a contrastarlas con el texto de Noctes Atticae 20.1.12, el cual citó en la forma "SI INIURIAM

\footnotetext{
76 Mommsen (1890) p. 145.

77 Mommsen (1890) p. 145.

78 Véase Mommsen (1890) pp. 109 y 110 y n. 1.; Volterra, E. (1930): "Collatio Legum Mosaicarum et Romanarum”, Memorie dell R. Accademia Nazionale dei Lincei. Classe di Scienze Morali, Storiche e Filologiche, Serie VI. - Volume III. - Fascicolo I. (Roma, Dott. Giovanni Bardi) pp. 8 a 23.

79 Pithoeus, P. (1656): Mosaycarum et Romanarum legum collatio... (Heidelbergae, Ex Typographeio Samuelis Broun) p. 129.
} 
ALTERI FAXIT"80. Así quedó trabado bajo el signo del acusativo "iniuriam"-, el círculo vicioso en la historia textual al que hicimos referencia.

Con los datos que hoy tenemos, el razonamiento debería ser exactamente el contrario. Las Noctes Atticae son una obra más antigua que la Collatio, por lo tanto, su testimonio de la Ley de las XII Tablas debería ser el más fiable -de lo cual hay, además, una ulterior prueba concreta, si se considera que Gelio da cuenta de las penas decenvirales en "ases", mientras Paulo, en la Collatio, incurre en el error de reportarlas en "sestercios" 81 , además del uso geliano del imperativo "sunto" para establecer la pena, que parece más correcto que el indicativo "subit" 82 ; de modo que si los códices de la obra de Gelio se inclinan por el ablativo adverbial “iniuria”, es más probable que este sea el contenido genuino; especialmente si, ahora para la Collatio, desde 1822 el códice de Vercelli también atestigua el mismo término "iniuria" en la cita de la norma decenviral.

Por otra parte, desde el punto de vista jurídico la lección "iniuriam-faxsit" (hace-injuria) es algo más fácilmente comprensible que "iniuria-faxsit" (injustamente-hace), pues el sentido de "hacer una injuria (iniuriam)", si bien no está exento de problemas, es más claro que el críptico y vago "injustamente (iniuria)-hacer" ${ }^{33}$, de

80 Pithoeus (1656) p. 130.

81 VöLKL (1984) p. 170; CURsI (2002) p. 231 n. 37.

82 Simon (1965) pp. 132 ss.; Halpin (1976) pp. 345 y 346 .

83 Esto ha sido muy correctamente explicado por Albanese, por más que ha nosotros no nos persuada la proposición de corrección textual que hace respecto de "alteri". Pugliese prefiere el acusativo "iniuriam" con un argumento completamente forzado. Excluye el ablativo "iniuria" solo porque a él le parece incompatible con el dativo "alteri" -que exigiría el acusati- cara a la reconstrucción de un precepto ( $\mathrm{Ta}$ bla 8.4) que se presume autónomo y conciso -en suma, con la corrección el precepto termina por no decir nada en sí- ${ }^{84}$. Sin embargo, ya Huvelin ${ }^{85}$ propuso el cambio de “iniuriam" por "iniuria” y, dada la adhesión suscitada ${ }^{86}$-pues esto incluso fue aceptado para la Collatio, por $S c h u l z^{87}$ y para la edición de la XII Tablas por Girard-Senn ${ }^{88}$-, obliga a replantearse el significado de "iniuria" en el texto de la Tabla $8.4^{89}$.

vo-; lo cual carece de todo fundamento, además de apoyarse en un término, "alteri", que ni siquiera se da por seguro en todas las ediciones de la norma. Cfr. Pugliese (1940) p. 2 n. 1; Albanese (1980) pp. 29 y 30; Cursi (2002) pp. 232.

$84 \mathrm{La}$ diferencia es tan radical, que no se puede aceptar posturas como las de Zimmermann, quien expone el problema de la diversidad de lecciones, pero termina por resolver la cuestión del modo tradicional (como si las fuentes simplemente dijeran "iniuriam"), sin haber resuelto en absoluto la cuestión. Tampoco parece adecuada la posición de Santalucia, quien desecha la lección con "iniuria" simplemente porque "ciò rende arduo determinare la portata della legge". Zimmermann (1996) pp. 1050 a 1053; SANTAlUCia $\left(1998^{2}\right)$ p. 61 n. 49.

85 Huvelin (1903) pp. 91 ss. y 109.

86 Schulz, F. (1951): "The manuscripts of the 'Collatio Legum Mosaicarum et Romanarum', BIDR 55-56, pp. 51 ss.; Birks (1969) pp. 164, 188 y 189; Wittmann (1972) pp. 9 ss.; Halpin (1976) p. 344-354; MANFredini (1977) pp. 26 ss. y 78 ss.; Albanese (1980) pp. 21 ss.; Völkl (1984) pp. 169 ss.; Westbrook, R. (1988): "Nature and origins of the Twelve Tables”, ZSS 105, pp. 103 ss.; Bassanelli Sommariva (1995) pp. 793-816; Cursi (2002) pp. 235 a 237; Cursi (2010) pp. 7 a 19; Bassanelli Sommariva (2012) pp. 260 a 268.

87 Schulz (1951) pp. 51 ss.

88 Girard; Senn et alii (1977) p. 63. Antes de esta edición, la obra de Girard no adoptaba la lección en comento, sino que reportaba como Tabla 8.4: SI INJURIAM [ALTERI] FAXSIT VIGINTI QUINQUE POENAE SUNTO. Cfr. GiRARD, P. (189512): Textes de droit romain (Paris, Arthur Rousseau) p. 16.

89 No ha tenido acogida la propuesta de Albanese, en cuanto a cambiar "alteri" por "alteras" o "aliter". Tampoco la de Manfredini, que propone sustituir 
3. Un tercer problema, relacionado en parte con lo anteriormente dicho, es determinar si en realidad puede dividirse el texto decenviral en tres preceptos autónomos (Tabla 8.2, 3 y 4).

Por una parte, todas las fuentes de transmisión nos muestran la norma sobre la rotura de un miembro, la fractura de un hueso y la iniuria como un conjunto. Por lo tanto, nada pareciera autorizar su interpretación separada, especialmente respecto de quienes han conjeturado que la Tabla 8.4 podría haber aparecido con posterioridad a las demás. En esto, además de la razón antedicha, es importante la lección "vinginti quinque poenae” entregada por Festo; pues su propia estructura deja implícito el medio de cambio en el que consistía, de lo cual resulta obvia la dependencia de la Tabla 8.4 respecto, al menos de la 8.3 , y de aquí una prueba de la necesaria interpretación unitaria del conjunto de los preceptos ${ }^{90}$.

Pero, además, debe considerarse aquí un texto que los editores han desechado en la reconstrucción de las Tablas 8.2 y 3: la obra de Catón el Viejo, Origines 4, que nos ha llegado a través de Prisciano, Institutiones Grammaticae 6.13.6991:

\footnotetext{
"facere" por "verberare". MANFREDINI (1977) pp. 78 a 84; Albanese (1980) pp. 21-36.

90 En cualquier caso, Albanese sostiene que tanto la lógica interna de la secuencia de preceptos de la Tabla 8.2, 3 y 4, como su exposición consistentemente conjunta en todas las fuentes por las cuales tenemos noticias de ellos, dan cuenta de que se trata de un cuerpo inescindible. Véase, Albanese (1980) pp. 30 y 31.

91 Cfr. Hertz, M. (1961): Prisciani Grammatici Caesarensis Institutionum Grammaticarum Libri XVIII, ex recensione Martini Hertzii, Vol. I. Libros I-XII continens, en Grammatici Latini ex recensione Henrici Keilii, Vol. II. Prisciani Institutionum Grammaticarum Libri I-XII ex recensione Martini Hertzii (Hildesheim, Georg Olms Verlagsbuchhandlung, pero, Leipzig, 1855) p. 254.
}

Cato tamen 'os' protulit in IIII originum: si quis membrum rupit aut os fregit, talione proximus cognatus ulciscitur...

(Sin embargo, Catón citó 'hueso' en su libro IV de Orígenes: si alguno rompió un miembro o fracturó un hueso, el cognado próximo se venga por el talión...)

En efecto, el testimonio de Catón une las Tablas 8.2 y 3; por cuanto la membri ruptio y la ossis fractio se subsumen bajo la común sanción del talión. A nuestro juicio, no puede desecharse con facilidad esta fuente, pues si bien ni Catón ni Prisciano fueron juristas -lo que obliga igualmente a tomarlo con cautela- ${ }^{92}$, este testimonio indirecto tiene la virtud de ser el único anterior a la lex Aebutia y, por tanto, correspondiente al momento en que las disposiciones decenvirales todavía se encontraban en vigor ${ }^{93}$.

92 Lo mismo en la obra sobre agricultura, que sin duda es la que ha concitado mayor atención de los romanistas, Castresana, A. (2009): Marco Porcio Catón. De agri cultura. Estudio preliminar, traducción $y$ notas (Madrid, Tecnos) p. LXXXVIII.

93 Así se atestigua por Aulo Gelio, Noctes Atticae 10.6.8: "Sed enim cum proletarii et adssidui et sanates et vades et subvades et viginti quinque asses et taliones furtorumque quaestio cum lance et licio evanuerint omnisque illa XII tabularum antiquitas, nisi in legis actionibus centumviralium causarum, lege Aebutia lata consopita sit, studium scientiamque ego praestare debeo iuris et legum vocumque earum quibus utimur" (Pero ciertamente como desaparecieron los proletarios y los asiduos y los sanates y los vades y subvades y los veinticinco ases y los taliones y los juicios de los hurtos por "lance y licio", y toda aquella antigüedad de las XII Tablas fuera abandonada por la promulgación de la ley Ebucia, salvo en las acciones de la ley de las causas centunvirales, yo debo servir el estudio y la ciencia del derecho y de los vocablos que usamos). Manfredini, señala que el testimonio de Catón corresponde a la disposición decenviral auténtica -no siempre con argumentos persuasivos, pero al menos en esto, creemos, está en lo correcto-. Manfredini (1977) pp. 72 ss. Contra, Pugliese (1978) pp. 195 y 196; SantaluCIA $\left(1998^{2}\right)$ p. 2 n. 2 y p. 61 n. 48. 